\title{
Tumors of the gastroesophageal junction have intermediate prognosis compared to tumors of the esophagus and stomach, but share the same clinical determinants
}

\author{
PAOLO G. GOBBI ${ }^{1}$, MANUELA BERGONZI ${ }^{1}$, DONATELLA POZZOLI ${ }^{1}$, LARA VILLANO ${ }^{1}$, \\ ALESSANDRO VANOLI $^{2}$, FRANCO CORBELLA ${ }^{3}$, PAOLO DIONIGI ${ }^{4}$ and GINO R. CORAZZA ${ }^{1}$
}

\begin{abstract}
${ }^{1}$ Medicina Interna e Gastroenterologia; ${ }^{2}$ Dipartimento di Patologia Umana ed Ereditaria, Sezione di Anatomia Patologica; ${ }^{3}$ Dipartimento di Onco-Ematologia, Sezione di Radioterapia Oncologica; ${ }^{4}$ Dipartimento di Scienze Chirurgiche, Sezione di Chirurgia Generale, Università di Pavia, Fondazione IRCCS Policlinico S. Matteo, Pavia 27100, Italy
\end{abstract}

Received November 24, 2010; Accepted February 21, 2011

DOI: $10.3892 / \mathrm{ol} .2011 .278$

\begin{abstract}
The issue of whether carcinoma of the gastroesophageal junction (GEJ) should be considered a distal esophageal, a proximal gastric or an independent tumor, at least with regards to clinical evaluation and management remains controversial. This study included 613 retrospective consecutive patients with carcinoma of the upper digestive tract, 64 of the esophagus, 58 of the GEJ and 491 of the stomach. The prognostic impact of the main clinical and histological parameters was analyzed in relation to relative survival as an estimate of the excess mortality. Relative survival and standardized mortality ratio (SMR) were calculated from the observed survival and the expected survival of the general population with identical age, gender and calendar years of observation. Multivariate analyses were applied to the proportional hazards model of the relative survival. The excess mortality, expressed by the relative survival and SMR of the patients with GEJ carcinoma are intermediate compared to those of patients with esophageal and gastric tumors. However, prognosis is not determined by tumor location, histology or administration of adjuvant chemotherapy, but mainly by stage and radical surgical resection. Gender has a minor but significant prognostic effect and age showed a slight inverse correlation with excess mortality. In conclusion, the excess mortality related to the tumors of the upper digestive tract is determined by stage, radical resection, gender and age. The intermediate prognosis of GEJ tumors mainly depends on a particular combination of such elementary determinants.
\end{abstract}

Correspondence to: Professor Paolo G. Gobbi, Medicina Interna e Gastroenterologia, Università di Pavia, Fondazione IRCCS Policlinico S. Matteo, P.le Golgi no. 2, 27100 Pavia, Italy

E-mail: gobbipg@smatteo.pv.it

Key words: gastroesophageal junction, cancer, prognosis, relative survival

\section{Introduction}

Topics such as the pathogenesis, clinical evaluation and treatment of tumors of the gastroesophageal junction (GEJ) are a controversial issue. This matter is likely due to the lower incidence of these tumors as compared to that of cancers of the esophagus or stomach, and to the fact that in clinical trials such tumors have been never treated as a distinct disease entity, but are generally grouped together with either esophageal or gastric cancers. Therefore, whether cancers of the esophagus, GEJ and stomach are a single disease, two diseases or more remains to be clarified (1).

In western countries, there is a trend to an increasing incidence of esophageal cancers as more of them are distal and of gastric cancers as more of them are proximal (2). In accordance with this epidemiologic shift of the carcinomas of contiguous digestive sections, the incidence of GEJ tumors was reported to have increased by 5 -fold over the last 20 years of the previous century (3). A recent worldwide overview documented a roughly homogeneous and steady incidence of cardia cancers since 1980, despite the declining incidence of other non-cardia carcinomas (4). However, the identification of GEJ does not rely on universally accepted criteria $(5,6)$. Staging classification varies for esophageal and gastric cancers and it is uncertain which staging is more appropriate for GEJ cancers. Appropriate staging is crucial since the clinical presentation of GEJ tumors is often locally advanced, showing spreading potentiality to both mediastinal and abdominal lymph nodes. Clinical investigations are currently ongoing. This study offers a clinical and prognostic evaluation of a series of GEJ carcinomas studied in a single institution during the last 25 years compared to the carcinomas of the esophagus and stomach diagnosed in the same period.

\section{Patients and methods}

Patients. Between January 1984 and December 2008, a consecutive series of 613 patients with carcinoma of the upper digestive tract were studied and treated in our Division. Of these, 64 patients presented with carcinoma of the esophagus, 
58 of the GEJ and 491 of the stomach. The large majority of these patients underwent surgical resection with radical intent in the Surgical Department of the Fondazione IRCCS Policlinico S. Matteo of Pavia, Italy. In particular, 39/64 patients were operated with tumors of the esophagus, 56/58 with GEJ tumors and $462 / 491$ with gastric tumors. The patients were followed up at the Department of Internal Medicine of the same Hospital until 31 December 2009. The data collected for each patient included: presenting signs and symptoms, tumor location, analytical description of the surgical procedures, radicality of the resection performed, macroscopic features of the resected tumor, diameters of the tumoral mass, number of regional metastatic lymph nodes, microscopic subtype of the tumor (presence or absence of distinct squamoid differentiation), Lauren's histological type (intestinal or diffuse), depth of penetration into the bowel wall, cell differentiation (well, moderately or poorly differentiated), grade of lymphatic invasion and main laboratory data at presentation prior to surgery (blood cell count, serum protein electrophoresis, liver and kidney function tests, and tumoral markers).

Macroscopic evaluation and description of the entire resected material and histological examination of the sampled specimens were systematically performed centrally. Vascular and lymphatic invasion was evaluated on paraffin sections stained with H\&E, while cases in which identification of endothelial structures was uncertain underwent immunohistochemical studies for CD34 and CD31 markers. The anti-CD31 antibody, which identifies the antigen ER-MP12, identical to the vascular endothelial adhesion molecule PECAM-1, and the anti-CD34 antibody, which stains normal and endothelial cells, make the identification of vascular and lymphatic vessels more straightforward. Systematic re-examination of the specimens was carried out to verify the correct diagnostic allocation into according to the categories of the WHO classification (7).

For the purposes of this study, patients alive in 2009 who had not undergone a medical examination within the preceding 6 months were recalled for a new clinical and instrumental control. The vital status of those patients who did not respond to this recall was ascertained by telephone (information from relatives and/or from physician) or investigated in the General Registry Offices of their last known municipality of residence. The patients were staged according to the last tumor, node and metastasis (TNM) classification (8), which stages tumors arising at the GEJ using the same criteria as those applied to esophageal neoplasias. These criteria include grading of cytologic differentiation, fix sharp limits for the discrimination of tumor location, adopt modified $\mathrm{T}, \mathrm{N}$ and $\mathrm{M}$ categories and, use separate classifications for squamous cell carcinoma and adenocarcinoma.

The anatomical discrimination of Siewert (9), substantially accepted in the recent version of the TNM classification, was used for the identification of GEJ tumors. In particular, type I tumors arise in the distal esophagus $1-5 \mathrm{~cm}$ from GEJ, type II is the true junctional tumors arising within $1 \mathrm{~cm}$ proximal and $2 \mathrm{~cm}$ distal to GEJ, and type III tumors are located from 2 to $5 \mathrm{~cm}$ distal to GEJ. Patient characteristics are shown in Table I.

Supportive therapy alone, without any antitumoral treatment, was administered to 10 patients with gastric tumors and to 2 patients with GEJ tumors, due to wide disease dissemina-
Table I. Main clinical characteristics of the study population related to the tumor location (percentages in brackets).

\begin{tabular}{|c|c|c|c|}
\hline & Esophagus & GEJ & Stomach \\
\hline Total no. & 64 & 58 & 491 \\
\hline \multicolumn{4}{|l|}{$\mathrm{M} / \mathrm{F}$} \\
\hline No. & $53 / 11$ & $37 / 21$ & $284 / 207$ \\
\hline Ratio & 4.82 & 1.76 & 1.37 \\
\hline \multicolumn{4}{|l|}{ Age (years) } \\
\hline Median & 64.3 & 65.3 & 64.1 \\
\hline Range & $43-93$ & $43-84$ & $19-91$ \\
\hline \multicolumn{4}{|l|}{ Histology } \\
\hline $\begin{array}{l}\text { Squamous or } \\
\text { adenosquamous }\end{array}$ & $54(84)$ & $3(5)$ & $1(0.002)$ \\
\hline \multicolumn{4}{|l|}{ Stage } \\
\hline 0 & 0 & 0 & $15(3)$ \\
\hline I A & 0 & 0 & $58(12)$ \\
\hline I B & 0 & $1(2)$ & $57(12)$ \\
\hline II A & $5(8)$ & $1(2)$ & $61(12)$ \\
\hline II B & $2(3)$ & $2(3)$ & $53(11)$ \\
\hline III A & $3(5)$ & $5(9)$ & $40(8)$ \\
\hline III B & $15(23)$ & $12(21)$ & $52(11)$ \\
\hline III C & $10(16)$ & $12(21)$ & $36(7)$ \\
\hline IV & $29(45)$ & $25(43)$ & $119(24)$ \\
\hline
\end{tabular}

tion and/or poor performance status, or heavy comorbidity. Surgical resection was performed in $61 \%$ of the esophageal, $97 \%$ of GEJ and in $94 \%$ of gastric tumors. Radical resection was performed in 80 of esophageal, 70 of GEJ and $80 \%$ of gastric tumors, respectively. Surgery was the only antitumoral treatment administered in 5 cases of esophageal, in 8 of cardial and in 132 of gastric tumor.

Adjuvant chemotherapy was administered to 41 patients with esophageal, 48 with GEJ and 202 with gastric tumor.

Radiotherapy was administered to 26 cases of esophageal tumor (in 7 cases it was the only antitumoral treatment adopted), while it was delivered in combination with chemotherapy in 2 cases of tumor of the GEJ and in 16 of the stomach. Table II shows the treatments administered, together with the chemotherapy regimens most frequently utilized.

The median follow-up was 27 months on the whole population (range 1-312), and 145 months for patients alive.

Statistical analysis. The time parameters taken into account were observed survival and relative survival. The latter was calculated as the ratio of the survival rate observed in the patients to the expected survival rate drawn from the general reference population for subjects similar to patients with respect to age, gender, calendar year of initial observation and duration of observation (10). The age-, gender- and calendar year-specific death rates available from the national Italian mortality tables (ISTAT, Istituto Nazionale di Statistica) were used to calculate the expected deaths and, thus, the expected survival. Age changes according to individual birthdays in every year of the follow-up were taken into account. Thus, a large control group was ensured from the 
Table II. Treatments adopted and chemotherapy regimens delivered to patients according to the tumor site (percentages in brackets).

\begin{tabular}{lccc}
\hline & Esophagus & GEJ & Stomach \\
\hline Operated & $39(61)$ & $56(97)$ & $462(94)$ \\
Radical resection & $31(80)$ & $40(70)$ & $368(80)$ \\
Radiotherapy & $26(40)$ & $2(4)$ & $16(3)$ \\
Chemotherapy & $41(64)$ & $48(83)$ & $202(41)$ \\
Type of chemotherapy & & & \\
CDDP, LF, FU & $29(71)$ & - & - \\
CDDP, EPI, LF, FU & $4(10)$ & $28(48)$ & $114(56)$ \\
LF, FU & $5(12)$ & $17(30)$ & $74(37)$ \\
CDDP, CPT-11 & $3(7)$ & $3(6)$ & $9(4)$ \\
OXA, LF, FU & - & - & $5(2)$ \\
\hline
\end{tabular}

CDDP, cisplatin; LF, leucovorin factor; FU, fluorouracil; EPI, epirubicin; CPT-11, irinotecan and OXA, oxaliplatin.

general population with corresponding personal characteristics and with a well-defined probability of succumbing to the disease. Consequently, the relative survival, obtained by adjusting observed survival for normal life expectancy, is considered to be a satisfactory estimate of the possibility of surviving the effects of cancer. A detailed example of the calculations required for each patient was provided elsewhere, in a study of a population with colorectal cancer (11). The observed deaths recorded in the patient population at the end of the follow-up period and the difference between the observed deaths and the cumulative expected probability of death during the corresponding period (i.e., excess mortality, which has to be taken into account for relative survival) are the data used in both survival calculations and multivariate analyses. The ratio of observed to expected deaths was used to determine the standardized mortality ratio (SMR).

The Kaplan-Meier method (12) was used to evaluate survival, and differences were analyzed by the log-rank test (13). The clinical and pathological characteristics that showed statistically significant prognostic values in univariate analyses were selected for multivariate analyses. The latter were performed by multiple regressions applied to a Cox proportional hazards model (14). A stepwise selection of factors was applied to the multiple regressions.

\section{Results}

The series of 613 patients with carcinoma of the upper digestive tract included 64 carcinomas of the esophagus, 58 of the GEJ, and 491 of the stomach. The data shown in Table I suggest that the age at onset of the three tumors is not different, while the gender ratio and prevalence of carcinoma with squamoid differentiation decrease as most distal is the location of the tumor. Notably, the anatomic and prognostic classification at diagnosis showed a prevalence of advanced stages (i.e., stages III and IV) among the esophageal (89\%) and GEJ tumors (94\%), whereas advanced presentation showed $50 \%$ of the gastric tumors.

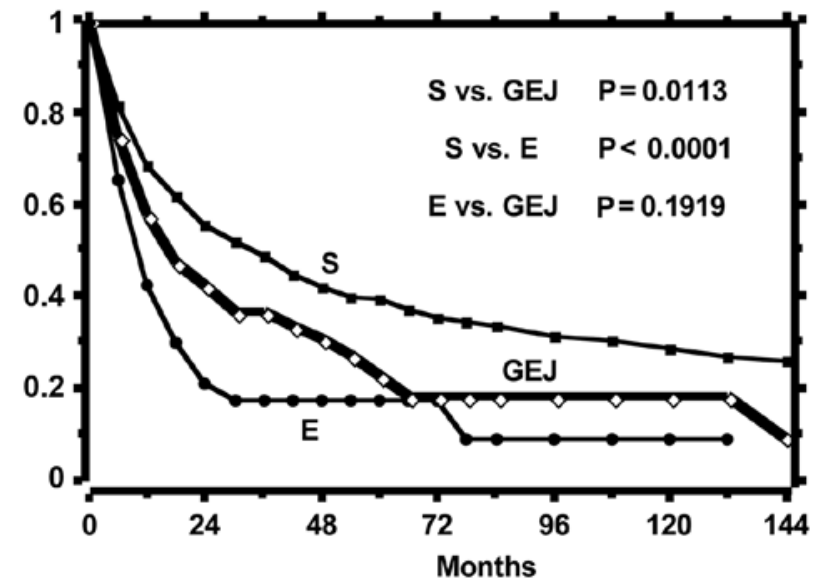

Figure 1. Observed survival of the patients according to the presentation site of the cancer (esophagus, GEJ and stomach).

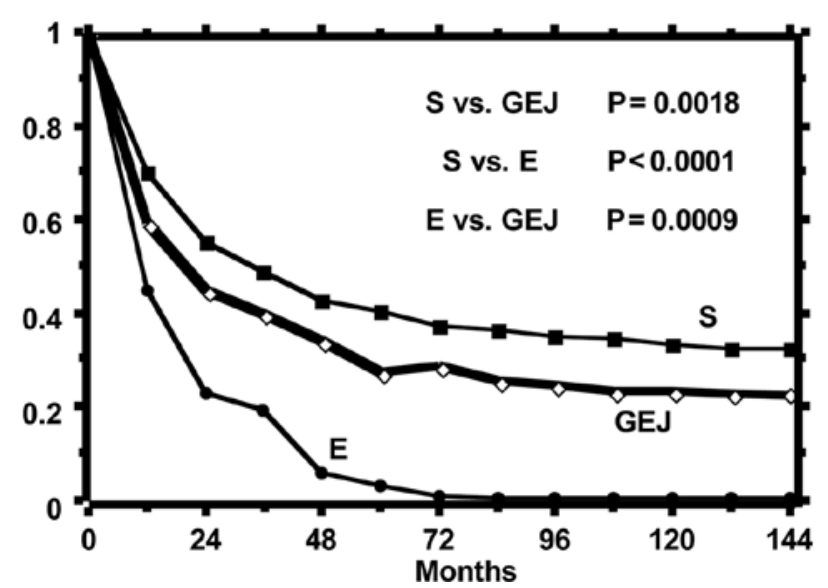

Figure 2. Relative survival of the same patient groups as Fig. 1. The curves indicate the excess mortality related to the mortality of the reference population: excess mortality is thought to be visually expresssed by the distance of each curve from a hypothetical horizontal straight line drawn at level 1 , corresponding to a null excess mortality (i.e., when the observed/expected survival ratio is equal to 1 ).

The curves of the observed and relative survival of the three groups of patients are shown in Figs. 1 and 2, respectively. Observed survival shows a statistically significant difference between gastric tumor and both esophageal and GEJ tumors, while the relative survival curves show a reduction of life expectancy statistically different among all groups. The highest excess mortality is noted in the esophageal tumor, while that of GEJ tumor is intermediate as compared to that of the esophagus and stomach. The SMR, distinct per site of tumor origin in the digestive tract, is reported in Table III, which shows that the mortality of gastric tumor is approximately 5-fold that of the normal reference population, that of the tumor of the GEJ is approximately 3-fold that of the stomach and, in turn, that of the esophageal tumor is twice that of the GEJ tumor. Minor differences in the SMR are observed within the three anatomical levels of origin of the esophageal tumors and among the three Siewert classes of the GEJ tumors.

A multivariate analysis of the relative survival was performed based on the clinical covariates of age, gender, 
Table III. Standardized mortality ratio (SMR) of the tumors of the esophagus, GEJ and stomach with further distinction related to the three levels of origin for the esophageal tumors and to the three Siewert's classes of the GEJ tumors.

\begin{tabular}{|c|c|c|c|c|c|c|c|}
\hline & \multicolumn{3}{|c|}{ Esophagus } & \multicolumn{3}{|c|}{ GEJ } & \multirow[t]{3}{*}{ Stomach } \\
\hline & \multirow{2}{*}{$\begin{array}{c}\text { Upper } \\
1 / 3\end{array}$} & \multirow{2}{*}{$\begin{array}{c}\text { Middle } \\
1 / 3\end{array}$} & \multirow{2}{*}{$\begin{array}{c}\text { Lower } \\
1 / 3\end{array}$} & \multicolumn{3}{|c|}{ Siewert's class } & \\
\hline & & & & I & II & III & \\
\hline No. & 17.0 & 27.0 & 20.0 & 10.0 & 36.0 & 12.0 & 491 \\
\hline SMR & 42.5 & 39.8 & 37.4 & 18.1 & 12.6 & 17.1 & 5.3 \\
\hline No. & & 64.0 & & & 58.0 & & 491 \\
\hline SMR & & 39.2 & & & 14.9 & & 5.3 \\
\hline
\end{tabular}

Table IV. Multivariate analysis according to the proportional hazard model applied to the relative survival of the 613 patients studied.

\begin{tabular}{lcccr}
\hline & Coefficient & $\begin{array}{c}\text { Standard } \\
\text { error }\end{array}$ & Chi-square & P-value \\
\hline Stage & 0.713 & 0.085 & 70.444 & $<0.0001$ \\
Radicality & 0.985 & 0.165 & 35.556 & $<0.0001$ \\
Age & -0.027 & 0.006 & 22.375 & $<0.0001$ \\
Gender & 0.409 & 0.139 & 8.711 & 0.0032 \\
\hline $\begin{array}{l}\text { Results of the backward selection of the best covariates (statistical } \\
\text { probability <0.05). The parameters considered during the initial step }\end{array}$ \\
$\begin{array}{l}\text { were gender, age, clinical stage, histology (squamous cell carcinoma } \\
\text { or otherwise), radicality of the surgical resection and original site of } \\
\text { the tumor (esophagus, GEJ or stomach). }\end{array}$
\end{tabular}

stage (the five categories of the seventh AJCC classification), histology (presence vs. absence of squamoid differentiation), radicality of the resection (yes vs. no), chemotherapy (yes vs. no) and site of tumor origin (esophagus vs. GEJ vs. stomach). The final results of the stepwise selection of the best covariates are reported in Table IV. Tumor location, histology and administration of chemotherapy do not significantly affect the reduction of life expectancy, whereas stage, radical resection, age and gender play a role in the reduction of life expectancy. Stage and gender exhibit a substantially unbalanced distribution among the three types of tumors, whereas age at diagnosis, which is relatively similar in the three tumors, has a limited but inversely proportional impact on relative survival, as noted by the absolutely low value and the negative sign of its coefficient.

\section{Discussion}

In view of the number of patients analyzed and the retrospective character of the study, firm conclusions cannot be drawn. However, the long follow-up period of time, the absence of selection biases in the series studied (including cases with any age at diagnosis, as well as the inoperable cases), and the analysis of relative survival for a more accurate prognostic evaluation suggest certain clinical observations. In particular, the choice of analyzing relative survival as the best estimate of specific survival and expression of the excess mortality due to the disease, is particularly suitable for patients that have i) a wide age range at diagnosis, including elderly subjects, and ii) a relatively long survival, and for these reasons are exposed to a number of factors, such as co-morbidities, accidents or complications, which compete with the tumor to reduce the life expectancy.

Since the relative survival is inferred from the expected survival from nationwide population life tables, stratified by age, gender and calendar time, a direct consequence of its consideration is the strong reduction of the prognostic importance of age, up to the inversion of its correlation with survival because of the correct weighing of the mortality due to co-morbidity at various ages. In this series, the age distribution at diagnosis did not present differences in the three cancer groups, but the excess mortality showed a weak but inverse correlation with age (the higher the age, the lower the excess mortality due to the tumor).

The main conclusion from this study regarding the excess mortality is that tumor location, histologic type and administration of chemotherapy lose any prognostic weight when analyzed with stage, radical resection, gender and age. As regards the histology, it should be noted that the well-known different pathogenesis of squamous cell carcinomas and adenocarcinomas is not under investigation. However, we show that the two carcinomas share a common prognosis that is secondary to other clinical factors. Moreover, the prognosis of the GEJ tumors, intermediate as compared to that of the esophageal and gastric ones, appears to be due not to distinct clinical features associated with the specific anatomic site, but with a combination of clinical factors that are common to the tumors of the contiguous regions.

Gender is a parameter showing a different distribution at presentation among the three tumors and may be involved in the prognostic grading generally observed in the moving from esophagus to stomach. However, the most significant factors are stage and radical resection. The distribution of these factors along the whole patient series elucidates the variation of life expectancy in a more favorable manner than tumor location, its histologic discrimination and the administration of chemotherapy.

This may mean that prognostic differences among tumors of the upper digestive tract are more related to the possible reasons for an advanced stage presentation and consequent difficulties in surgical management, such as conditions 
for delayed diagnosis, different opportunity of anatomic spreading and different possibility of radical resection. This does not necessarily mean we are dealing with the same type of tumor arising from esophagus to stomach, since some distinctive characteristics related to distribution of histological type, gender ratio and pathogenetic mechanisms are clear. Our data suggest that these differences are less important than stage and radical resection when correlated with prognosis.

In this regard, the evaluation of the SMR appears to indicate that the subdivision of the GEJ tumor into the three classes identified by Siewert is less justified, since it was not regarded as significant that a cardiac tumor develop within $5 \mathrm{~cm}$ upward or downward of the GEJ. The significant factor is the origin from GEJ, a site which shares the mentioned probabilities of delayed diagnosis and advanced stage at presentation, of wide pre-clinical spread and consequently more complex surgical needs. This concept substantially agrees with the conclusions of Jin et al (15) and of Maeda et al (16), but further confirmation is required.

\section{Acknowledgements}

This study was supported in part by grants from the Fondazione IRCCS Policlinico S. Matteo, Pavia, and the 'Ferrata-Storti Foundation', Pavia. We are indebted to Dr Rachel Stenner for the revision of the English of this paper.

\section{References}

1. Rusch VW: Are cancers of the esophagus, gastroesophageal junction, and cardia one disease, two or several? Sem Oncol 31: 444-449, 2004.

2. Apisarnthanarax S and Tepper J: Crossroads in the combinedmodality management of gastroesophageal junction carcinomas. Gastrointest Cancer Res 2: 235-243, 2008.
3. Devesa SS, Blot WJ and Fraumeni J Jr: Changing patterns in the incidence of esophageal and gastric carcinoma in the United States. Cancer 83: 2049-2053, 1998.

4. Bertuccio P, Chatneoud L, Levi F, et al: Recent patterns in gastric cancer: a global overview. Int J Cancer 125: 666-673, 2009.

5. Sato T, Kato Y, Matsuura M and Gagner M: Significance of palisading longitudinal esophagus vessels: identification of the true esophagogastric junction has histopathological and oncological considerations. Dig Dis Sci 55: 3095-3101, 2010.

6. Takubo K, Vieth M, Aida J, Sawabe M, Kumagai Y, Hoshihara Y and Arai T: Differences in the definition used for esophageal and gastric disease in different countries. Digestion 80: 248-257, 2009.

7. Hamilton SR and Aaltonen LA: Pathology and Genetics. Tumors of the Digestive System. World Health Organisation Classification of Tumours, Lyon, IARC press, pp1-314, 2000.

8. AJCC (American Joint Committee on Cancer) Cancer Staging Manual. 7th edition. Edge SB, Byrd DR, Compton CC, et al (eds). Springer, New York, pp103-110 and 117-121, 2009

9. Siewert JR and Stein HJ: Classification of adenocarcinoma of the esophageal junction. Br J Surg 85: 1457-1459, 1998.

10. Armitage P and Berry G: Statistical Methods in Medical Research. 2nd Edition. Blackwell Scientific Publications, Oxford, pp403-405, 1987.

11. Gobbi PG, Valentino F, Berardi E, et al: New insights into the role of age and carcinoembryonic antigen in the prognosis of colorectal cancer. Br J Cancer 98: 328-334, 2008.

12. Kaplan EL and Meier P: Non parametric estimation from incomplete observations. J Am Stat Assoc 53: 457-481, 1958.

13. Peto R, Pike MC, Armitage P, et al: Design and analysis of randomized clinical trials requiring prolonged observation of each patient. II. Analysis and examples. Br J Cancer 35: 1-39, 1977.

14. Cox DR: Regression models and life tables. J R Stat Soc 34: 187-220, 1972

15. Jin L, Yoshida M, Kilagawa Y, et al: Subclassification of superficial cardia cancer in relation to the endoscopic esophagogastric junction. J Gastroenterol Hepatol (Suppl 2): 273-277, 2008.

16. Maeda H, Okabayashi T, Nishimori I, et al: Clinicopathologic features of adenocarcinoma at the gastric cardia: is it different from distal cancer of the stomach? J Am Coll Surg 208: 308-310, 2008. 